\title{
Making healthcare decisions for terminally ill adults and elderly in rural Bangladesh: an application of social autopsy
}

\author{
Nurul Alam1', Hafizur Rahman Chowdhury², Sayed Saidul Alam¹, Taslim Ali', Peter Kim Streatfield', Ian Douglas Riley², \\ Alan D Lopez ${ }^{2}$ \\ 1 icddr,b, Health Systems and Population Studies Division, Mohakhali, Dhaka, Bangladesh, 2 University of Melbourne, School of Population and Global \\ Health, Carlton, Victoria, Australia \\ Keywords: bangladesh, social autopsy, global health \\ https://doi.org/10.29392/joghr.3.e2019002
}

\section{Journal of Global Health Reports}

Vol. 3, 2019

\begin{abstract}
Background
This study investigated healthcare decision-making surrounding terminal illnesses of adults and elderly persons (aged 15 years or more), who later died at home, hospitals, or in-transit, in a rural, low-income area of Bangladesh where out-of-pocket health expenditure is very high.
\end{abstract}

\begin{abstract}
Methods
There were 1330 adult and elderly deaths in Matlab Health and Demographic Surveillance site in 2013. To record how decisions were made and implemented when caring for terminal illness, and how treatment-related costs were managed, the main caregivers of 69 randomly selected persons who died at home, 74 who died in hospitals, and 11 who died in-transit were interviewed with a social autopsy questionnaire in 2014. Differences between groups were tested by $\chi^{2}$ for significance at $P<0.05$.
\end{abstract}

\begin{abstract}
Results
Those who died at home were sick for longer periods than those who died in hospitals or in-transit. During terminal illness, 33\% of the home deaths were admitted, but discharged from hospitals prior to death for no chance of cure (52\%) or financial constraints (17\%). The reasons for $67 \%$ of home deaths not being admitted to a hospital were no chance of cure (28\%), misjudgment of illness severity (21\%), too short duration of illness (20\%), or financial constraints (17\%). For hospital deaths, final decisions were mostly made by family members (95\%) as opposed to physicians (5\%) for cure of serious illness (93\%). Hospitals were chosen considering quality of care (59\%), distance (36\%), known doctors (35\%) or referral (29\%). After hospitals were chosen, $42 \%$ of hospitalizations were delayed due to lack of money (33\%), misjudgment of illness severity (31\%), or lack of someone to accompany the patient (31\%). Payment of hospital costs included family savings (87\%), borrowing from relatives (46\%) or borrowing from moneylenders (6\%).
\end{abstract}

\section{Conclusions}

Healthcare decisions for terminally ill adults and elderly persons were influenced by the caregivers' assessment of chances of cure, judgment of illness severity and financial constraints. Improving understanding of illness severity and lowering direct out-of-pocket expenditures may help patients who have a reasonable chance of cure receive care in a hospital.

Most of the literature on social autopsies of death or medical consultations prior to death of newborns and children focus on access to health services and the extent of service utilization as contributors to death. ${ }^{1,2}$ Decisions to do with caring for the ill person shape how health services of varying types, qualities and costs are utilized. Making decisions for treatment is a process of assessing risk and chance of cure; knowledge and weighing up of multiple options for treatment; and other aspects, including costs of medical care. ${ }^{3}$ Therefore, decision-making is an integral part of a patient's healthcare-seeking behaviour and constitutes a key component of healthcare improvement and reform. Little is known, however, how decision-making occurs in countries that lack risk-pooling mechanisms.

In many low and middle-income countries, including Bangladesh, the risk-pooling mechanisms (such as health insurance, community or group managed insurance, social safety net, etc.) are not functional, whilst out-of-pocket 
payments are the most common means of financing healthcare. ${ }^{4}$ In Bangladesh, average out-of-pocket healthcare expenditure is very high, comprising $65 \%$ of the total health expenditures, ${ }^{5}$ potentially creating financial hardship for lower-income groups in accessing quality services. Direct out-of-pocket payments at the household level at the time of need for care are identified as major barriers to accessing quality medical care for sick children. ${ }^{6}$ For elderly people, only a small fraction of deaths occurs in hospitals, potentially due to financial constraints and community perceptions related to care of the elderly. For example, deaths registered in a rural demographic surveillance site during 2003-04 showed that only $10 \%$ of adult and elderly deaths occurred in hospital, with $4 \%$ of those deaths being women and another $4 \%$ are elderly. ${ }^{7}$ Such a low proportion of deaths in hospitals might be the result of the household's response to the terminal illness, determined by chances of cure, severity of illness, financial restrictions, and availability of a person to accompany and provide care to the patient in hospital. No study has so far critically examined how decisions for treatment of severe illnesses of adults and elderly are made and implemented, and how treatmentrelated costs are managed in rural communities where household income is relatively low, and direct out-of-pocket payment at the time of care is dominant.

Adults and the elderly in Bangladesh mostly die from chronic and non-communicable diseases (NCDs). ${ }^{7,8}$ NCDs such as cancer, cardiovascular diseases, chronic respiratory diseases, diabetes, chronic kidney and liver diseases, etc. are tackled at secondary and tertiary levels, mainly through specialized facilities which have skilled health workers and sophisticated equipment for diagnosis, treatment and management. ${ }^{9}$ Medication and diagnostics, however, are often unavailable in these higher-level facilities. ${ }^{10}$ Moreover, public health facilities in rural areas mostly focused on infectious diseases with some attention given to screening for diabetes and hypertension. These facilities lack diagnostic capacity, medicines, and quality treatment for diabetes or cardiovascular diseases. ${ }^{11,12}$ A rural person suffering from NCDs must therefore visit a secondary- or tertiary-level hospital in a town or city for treatment, meaning that a family seeking medical care on behalf of an ill person needs to make three decisions: "where" to take the patient, "who" can accompany him/her to a distant hospital, and "how" financing can be managed.

Some NCDs require expensive treatment for a longer period. In Kenya, the high cost of NCD services relative to income makes NCD services unaffordable for most people, and eventually deters many people suffering from NCDs from seeking the care they need. ${ }^{13}$ In Dhaka, main household income earners (mostly males) living in urban slums face chronic incapacitating ill-health, which has serious effects on the ability of these households to pay for health services of optimum quality. ${ }^{14}$ Because of high out-ofpocket health expenditures and loss of wages, these affected households often must dispose of most of their assets to pay for medical treatment. ${ }^{14}$ In Lao People's Democratic Republic (PDR), decisions regarding care-seeking are constrained by high medical costs, low expectations of recovery and a perceived low quality of care at the health facilities. ${ }^{15}$ Therefore, a household facing multiple illness episodes over time may eventually lose economic capability to the point where choice of a treatment provider is compromised, and treatment is delayed.

Making decisions regarding the medical treatment (and associated finances) of critically ill adults and the elderly involve interactions between the patient, family members, relatives, neighbours and healthcare providers. Their decisions regarding health financing, treatment, and choice of healthcare providers/facilities are based on judgments of the severity of the illness and expectations of recovery. Physicians usually play a vital role in the decision-making process, particularly in making the final choice of a healthcare provider or facility. On the Kenyan coast, as fathers are the main income earners, they make the final decisions concerning where the child is taken for treatment. ${ }^{16}$ In Bangladesh, treatment-related costs are generally high, particularly in private sector facilities, and need to be paid up-front. Therefore, household members who have the ability and will to pay medical bills arguably make the final choice of treatment provider or facility, which can be different from the decision a physician makes.

Perceptions and attitudes to do with care and cure of illness prevalent in the family and community determine healthcare seeking for elderly people. They usually suffer from chronic NCDs and associated co-morbidities, some of which have no cure. A qualitative study in Bangladesh noted that 'responding to illness of the elderly people is rather resigned to the belief that "illness in old age is not completely curable and sometimes incurable if their illnesses do not respond to treatments". ${ }^{17}$ Community attitudes towards tackling illnesses of elderly people dictate that care should be given at home, with indigenous health providers, or with formally trained providers if the illness is severe.

For more understanding of the social processes of making and implementing decisions surrounding medical care seeking for terminally ill patients in a rural community, a study was designed in 2014 for obtaining social autopsies of a random sample of adult and elderly deaths that happened either at home, in health facilities or on the way to a health facility in Bangladesh in 2013. Therefore, the objectives of the study were to investigate how treatment decisions were made in the family, the reasons for deciding against taking patients to a hospital after recognizing illness, who made the final choice of treatment provider or facility, the reasons for delay in seeking treatment after a provider/facility was identified, and how healthcare expenditures were managed. The findings of the study may be used to fill the information gap in healthcare decision-making, identify areas for interventions to ensure care for patients who have reasonable chances of cure from well-trained health service providers, allow communities themselves to take local level actions, and contribute to national-level advocacy for greater accountability in health systems and healthcare financing.

\section{METHODS}

The Health and Demographic Surveillance System (HDSS) maintained by icddr,b (International Centre for Diarrhoeal Disease Research, Bangladesh) in Matlab, Bangladesh since 1966 covered a de-jure population of 227,853 in 142 villages in 2013. ${ }^{18}$ Matlab is one of the 490 rural sub-districts in 
Bangladesh, and is located $55 \mathrm{~km}$ southwest of the capital city of Dhaka. HDSS field workers visiting households bimonthly recorded 1330 deaths aged 15 years and above in 2013. Field supervisors were able to obtain verbal autopsy (VA) data, including place of death, for 1323 (99\%) out of 1330 deaths.

Place of death was home for 1127 deaths, health facilities for 138 deaths and on the way to a health facility for 58 deaths, totaling 1323 deaths with VA. In order to investigate the decision-making process for caring for adult and elderly terminally ill patients who later died at home, in health facilities or on the way to a health facility and for identifying family-level barriers in accessing healthcare a social autopsy survey was conducted in 2014 over 12 to 18 months after the date of death in 2013. Considering the overall objective of the survey and availability of resources, sample size per group (home or hospital as place of death) was estimated to be 67 so that parameters could be estimated with precision of $\pm 12.5 \%$ at $95 \%$ confidence interval. The sample size was further increased to 75 to adjust for non-response or absence of the main respondents on the survey date. The sample size for the in-transit deaths was 15. From three separate lists of deaths that occurred at home, hospitals or in-transit, required number of sampled deaths per group was randomly selected for the survey. The survey team consisting of HDSS field supervisors was able to obtain social autopsy of 69 home deaths, 74 hospital deaths and 11 intransit deaths, totaling 154 deaths.

The field supervisors were trained on how to identify the main caretakers of the deceased, obtain their consent and maintain privacy, anonymity and confidentiality of data/information related to the deceased. They were provided with a list of sampled deaths to interview the main caregivers at their homes using a social autopsy questionnaire. With the consent of caregivers, the field supervisors asked about discussions with family members, relatives and healthcare providers on illness severity, making the decisions for shifting or not shifting the patients to a hospital, reasons for deciding against taking a patient to a hospital, involvement of healthcare providers in making decisions and choice of a hospital. They also inquired about factors that resulted in shifting patients to hospitals, items considered in choosing a particular health provider or a hospital among alternatives, reasons for delays in care seeking, reasons for discharge from hospital prior to death and payment of the treatment costs.

Details of medical signs and symptoms or conditions that led to death were extracted from VAs obtained in 2013. A trained medical assistant (with three years training in medicine in public sector) reviewed each VA and assigned a possible underlying cause of death with ICD 10 code (ICD-10 Version: 2016). Cause of death was categorized into three broad groups; communicable diseases, non-communicable diseases (NCDs) and injury for small sample size.

A household socioeconomic census was conducted in 2014 to record possession of durable items. The principal components analysis of household durables retained one factor and assigned a factor score to each household. A higher score indicates a better long-term economic status of the household. The factor score was used to divide households into three equal groups (low, medium and high). Social autopsies of the deceased were linked with household asset score.

\section{DATA ANALYSIS}

Proportion for categorical variables and average for quantitative variables were used to show differences between two groups. Chi-square statistic was used to test the difference between proportions for significance set at $P<0.05$.

\section{ETHICS}

This survey was a part of the parent project entitled "Improving Method to Measure Comparable Mortality by Cause (PR \# 10086)"; approved by the Ethical Review Committee of icddr,b on the $23^{\text {rd }}$ December $2010 .{ }^{19}$

\section{RESULTS}

\section{COMPARISON OF ALL DEATHS AND SAMPLED DEATHS}

Age distributions of all deaths aged 15 years and above and the sampled deaths for social autopsy were similar, but highly dissimilar in distribution by place of death for oversampling from the list of hospital deaths (Table 1). As expected, the percentage of hospital deaths was much higher in the sample than it was in all deaths.

\section{DESCRIPTIVE STATISTICS OF THE SAMPLED DECEASED}

Sex distribution of the decedents who died at home and those who died out of home was comparable, but they were different in terms of age distribution (Table 2). The majority (75\%) of the decedents who died at home were elderly, as opposed to $45 \%$ of the decedents who died in hospitals. The decedents who died at home were on an average 12 years older than the decedents who died in hospitals. The average duration of illness prior to death was shorter for hospital or in-transit deaths than home deaths. While all the hospital deaths were visited by medical doctors during terminal illness, only $52 \%$ of the home deaths and $64 \%$ of the in-transit deaths were visited by medical doctors. Patients consulted both qualified and non-qualified providers of modern medicine and in some cases traditional providers to treat illness. The number of consultations with village doctors and other indigenous healthcare providers (such as herbalists or spiritual healers) was higher for the home and in-transit deaths. Both the percentage and mean times of hospitalization during terminal illness were lower for home and intransit deaths when compared to hospital deaths.

Socioeconomic circumstances of the deceased were measured by their level of education and household asset scores. The decedents who died at home had a lower education level and lower asset score, compared to those who died in hospitals or in-transit.

Distribution of deaths by cause shows that $85 \%$ of the deaths were due to NCDs, with a proportion being higher (93\%) in the elderly age group (Table 3). All injury deaths were taken to hospitals, compared to a half of the deaths caused by communicable diseases and NCDs. While NCD deaths were more common in the top tertile households, deaths due to communicable diseases and injury were more 
Table 1. Distribution of all adult and elderly deaths and sampled deaths for the social autopsy survey by age and place of death, HDSS 2013

\begin{tabular}{|c|c|c|c|c|c|c|}
\hline \multirow{2}{*}{$\begin{array}{l}\text { Age at death (in } \\
\text { years) }\end{array}$} & \multicolumn{3}{|c|}{ Number (and \%) of all deaths } & \multicolumn{3}{|c|}{ Number (and \%) of sampled deaths } \\
\hline & $\begin{array}{l}\text { Hospital or in } \\
\text { transit }\end{array}$ & At home & Total & $\begin{array}{l}\text { Hospital or in } \\
\text { transit }\end{array}$ & At home & Total \\
\hline Adult (aged 15-64) & $116(27 \%)$ & $\begin{array}{l}319 \\
(73 \%)\end{array}$ & $\begin{array}{c}435 \\
(100 \%)\end{array}$ & $46(73 \%)$ & $\begin{array}{c}17 \\
(27 \%)\end{array}$ & $\begin{array}{c}63 \\
(100 \%)\end{array}$ \\
\hline Elderly (aged 65+) & $80(9 \%)$ & $\begin{array}{l}808 \\
(91 \%)\end{array}$ & $\begin{array}{c}888 \\
(100 \%)\end{array}$ & $39(43 \%)$ & $\begin{array}{c}52 \\
(57 \%)\end{array}$ & $\begin{array}{c}91 \\
(100 \%)\end{array}$ \\
\hline Total* & $196(15 \%)$ & $\begin{array}{l}1127 \\
(85 \%)\end{array}$ & $\begin{array}{c}1323 \\
(100 \%)\end{array}$ & $85(55 \%)$ & $\begin{array}{c}69 \\
(45 \%)\end{array}$ & $\begin{array}{c}154 \\
(100 \%)\end{array}$ \\
\hline
\end{tabular}

*Verbal autopsy could not be obtained for 7 deaths and hence excluded from analysis.

Table 3. Causes of the sampled deaths by variables: age, sex, household asset tertile and place of death

\begin{tabular}{|c|c|c|c|c|c|}
\hline \multirow[b]{2}{*}{ Variable name } & \multicolumn{4}{|c|}{ Cause of death } & \multirow{2}{*}{$\begin{array}{c}\mathrm{X}^{2} \text { and } P \text {-value } \\
\text { (CD, NCD and injury) }\end{array}$} \\
\hline & $\begin{array}{l}\text { Communicable disease (CD) } \\
(n=16) \%\end{array}$ & $\begin{array}{l}\text { Non-CD } \\
(n=131) \%\end{array}$ & $\begin{array}{c}\text { Injury } \\
(n=7) \%\end{array}$ & $\begin{array}{c}\text { Total } \\
(n=154) \%\end{array}$ & \\
\hline \multicolumn{6}{|l|}{ Age at death: } \\
\hline $15-64$ years & 17.5 & 73.0 & 9.5 & $63(100.0)$ & \multirow[t]{2}{*}{$12.7, P=0.002$} \\
\hline $65+$ years & 5.5 & 93.4 & 1.1 & $91(100.0)$ & \\
\hline \multicolumn{5}{|l|}{ Sex of the deceased: } & \multirow{3}{*}{$1.5, P=0.46$} \\
\hline Male & 12.6 & 83.9 & 3.4 & $87(100.0)$ & \\
\hline Female & 7.5 & 86.6 & 16.0 & $67(100.0)$ & \\
\hline \multicolumn{5}{|c|}{ Household asset tertile: } & \multirow{4}{*}{$6.7, P=0.15$} \\
\hline Low & 14.6 & 78.0 & 7.3 & $41(100.0)$ & \\
\hline Medium & 14.3 & 78.6 & 7.1 & $42(100.0)$ & \\
\hline High & 5.6 & 93.0 & 1.4 & $71(100.0)$ & \\
\hline \multicolumn{5}{|l|}{ Place of death: } & \multirow{4}{*}{$6.0, P=0.05$} \\
\hline Home & 11.6 & 88.4 & 0.0 & $69(100.0)$ & \\
\hline Hospital/in transit & 9.4 & 82.3 & 8.2 & $85(100.0)$ & \\
\hline All & 10.4 & 85.1 & 4.6 & $154(100.0)$ & \\
\hline
\end{tabular}

$\mathrm{N}$-number of deaths in each category, NCD - non-communicable disease

common in the lower tertile households. However, this difference was not statistically significant.

Distribution of the relationships of the participants involved in discussions related to making treatment decisions for the deceased who died at home and who died in hospitals showed no difference (see Table 1a in Online Supplementary Document(Online Supplementary Document)). Participation of family members in the discussions was universal (>98\%). Among the family members involved to a large extent were sons and daughters (88\% and $77 \%$ respectively), followed by spouses (49\% and $53 \%$ respectively) for home and hospital deaths. The other common participants were relatives (51\%), followed by healthcare providers (30\%) and neighbours or friends (26\%).

Two-thirds of the 69 people who died at home were not admitted in any hospital, and the rest were admitted but discharged from hospitals during terminal illness.
PATIENT DEATHS AT HOME: REASONS FOR NOT TAKING TO HOSPITAL OR FOR DISCHARGING FROM A HOSPITAL

Discussion related to hospitalization during illness of the deceased in households was 62\% (Table 4). Rate of the discussion exhibited age and sex biases; it was higher for adult than for elderly patients and for male than for female patients. Reasons for deciding against taking the deceased to a hospital during illness and for discharging prior to death from the hospital are presented in Table 5. The most common reason stated was 'no chance of cure of illness' - this was higher for hospital discharged cases (52\% versus $28 \%$ ). The second most common reason was the inability to bear hospital and treatment costs (17\% each). Misjudgment of the severity of illness and too short duration of illness to decide and shift to a hospital were higher (22\% and 20\% respectively, versus $4 \%$ each) for non-hospitalized cases. The other, less common reasons were refusal by the patients to move (6\%), a lack of someone to accompany the patient to the hospital (6\%) and that the patient was not apparently sick (3\%). 
Table 2. Socio-demographic and illness characteristics of the sampled deaths by place of death.

\begin{tabular}{|c|c|c|c|c|c|}
\hline \multirow[t]{2}{*}{ Characteristics } & \multicolumn{4}{|c|}{ Place of death } & \multirow{2}{*}{$\begin{array}{c}\mathrm{X}^{2} \text { and } P \text {-value } \\
\text { (home, in transit and } \\
\text { hospital) }\end{array}$} \\
\hline & $\begin{array}{c}\text { Home } \\
(n=69) \%\end{array}$ & $\begin{array}{l}\text { In transit } \\
(n=11) \%\end{array}$ & $\begin{array}{l}\text { Hospital } \\
(n=74) \%\end{array}$ & $\begin{array}{c}\text { Total } \\
(n=154) \%\end{array}$ & \\
\hline \multicolumn{5}{|l|}{ Age at death (in years): } & \multirow{4}{*}{$14.1, P=0.001$} \\
\hline $15-64$ & 24.6 & 45.4 & 55.4 & 40.9 & \\
\hline $65+$ & 75.4 & 54.6 & 44.6 & 59.1 & \\
\hline Mean \pm SD (Median) & $\begin{array}{l}71.4 \pm 12.6 \\
(72)\end{array}$ & $\begin{array}{l}63.7 \pm 17.8 \\
(69)\end{array}$ & $\begin{array}{l}59.4 \pm 18.0 \\
(62.5)\end{array}$ & $\begin{array}{l}65.1 \pm 16.7 \\
(68)\end{array}$ & \\
\hline \multicolumn{5}{|l|}{ Sex of the deceased: } & \multirow{3}{*}{$0.1, P=0.99$} \\
\hline Male & 56.5 & 54.6 & 56.8 & 56.5 & \\
\hline Female & 43.5 & 45.4 & 43.2 & 43.5 & \\
\hline \multicolumn{5}{|c|}{ Duration of terminal illness:* } & \multirow{5}{*}{$6.1, P=0.05$} \\
\hline$<22$ days & 42.0 & 54.6 & 64.9 & 53.9 & \\
\hline $22+$ days & 55.1 & 36.4 & 31.1 & 42.2 & \\
\hline Missing & 2.9 & 9.1 & 4.0 & 3.9 & \\
\hline Mean \pm SD (Median) & $\begin{array}{c}183.8 \pm 374.5 \\
(60)\end{array}$ & $\begin{array}{l}71.2 \pm 119.1 \\
\text { (3) }\end{array}$ & $\begin{array}{l}39.3 \pm 80.2 \\
(8)\end{array}$ & $\begin{array}{c}106.9 \pm 268.1 \\
(12.5)\end{array}$ & \\
\hline \multicolumn{5}{|c|}{ Type of medical consultation prior to death: } & \\
\hline Medical doctor & 52.2 & 63.6 & 100.0 & 75.4 & 45.7, $P=0.001$ \\
\hline Paramedics/nurse & 10.1 & 18.2 & 8.1 & 9.7 & $1.1, P=0.57$ \\
\hline $\begin{array}{l}\text { Village (untrained) } \\
\text { doctor }\end{array}$ & 65.2 & 54.6 & 37.8 & 51.3 & $10.8, P=0.005$ \\
\hline Homeopath & 7.2 & 18.2 & 5.4 & 7.1 & $2.4, P=0.31$ \\
\hline Ayurveda (herbalist) & 17.4 & 9.1 & 5.4 & 11.0 & $5.3, P=0.07$ \\
\hline Spiritual & 13.0 & 0.0 & 2.7 & 7.4 & \multirow[t]{2}{*}{$6.7, P=0.04$} \\
\hline \multicolumn{5}{|c|}{ Hospital admission during terminal illness: } & \\
\hline Admitted in hospital & 33.3 & 45.4 & 100.0 & 66.2 & \multirow{2}{*}{ 73.2, $P=0.001$} \\
\hline $\begin{array}{l}\text { Times admitted (mean } \\
\pm \text { SD) }\end{array}$ & $0.6 \pm 1.0$ & $0.5 \pm 0.7$ & $1.7 \pm 0.9$ & $1.1 \pm 1.1$ & \\
\hline \multicolumn{5}{|l|}{ Level of education: } & \multirow{4}{*}{$10.7, P=0.03$} \\
\hline None & 60.9 & 45.5 & 33.8 & 46.7 & \\
\hline Primary & 17.4 & 27.3 & 32.4 & 25.3 & \\
\hline Secondary and above & 21.7 & 27.3 & 33.8 & 27.9 & \\
\hline \multicolumn{5}{|l|}{ Household asset tertile: } & \multirow{4}{*}{$16.7, P=0.002$} \\
\hline Low & 36.2 & 27.3 & 17.6 & 27.1 & \\
\hline Medium & 34.8 & 18.2 & 21.6 & 27.1 & \\
\hline High & 29.0 & 54.6 & 60.8 & 45.7 & \\
\hline
\end{tabular}

$\mathrm{N}$ - number of deaths in each category, SD - standard deviation

*No illness duration reported for 6 deaths; 4 died from ischemic heart disease and 2 from strokes.

Table 4. Discussion on hospitalization during terminal illness of the deceased who died at home by their age and sex

\begin{tabular}{cccccc}
\hline Age of the deceased & \% had discussion & $\begin{array}{c}\mathrm{X}^{2} \text { and } \\
\boldsymbol{P} \text {-value }\end{array}$ & Sex of the deceased & \% had discussion & $\begin{array}{c}\mathrm{X}^{2} \text { and } \\
\boldsymbol{P} \text {-value }\end{array}$ \\
\hline $15-64(\mathrm{n}=17)$ & 82.3 & $3.86, P=0.05$ & Male $(\mathrm{n}=39)$ & 71.8 & $3.43, P=0.06$ \\
$65+(\mathrm{n}=52)$ & 55.8 & & Female $(\mathrm{n}=30)$ & 50.0 & \\
All $(\mathrm{n}=69)$ & 62.3 & All $(\mathrm{n}=69)$ & 62.3 \\
\hline
\end{tabular}

$\mathrm{n}$ - number of deaths in each category 
Table 5. Reasons for not admitting in or discharging from hospitals during terminal illness of the deceased who died at home

\begin{tabular}{lccc}
\hline Reasons for not admitting or discharging & $\begin{array}{c}\text { Not admitted in hospital } \\
(\mathbf{n}=\mathbf{4 6 )} \%\end{array}$ & $\begin{array}{c}\text { Admitted, but discharged } \\
(\mathbf{n}=\mathbf{2 3}) \%\end{array}$ & $\begin{array}{c}\text { Total home deaths } \\
(\mathbf{n}=\mathbf{6 9}) \%\end{array}$ \\
\hline No hope for cure & 28.3 & 52.2 & 36.2 \\
Unable to bear cost & 17.4 & 17.4 & 17.4 \\
Misjudge severity of illness & 21.7 & 4.3 & 15.9 \\
Not enough time to decide and shift & 19.6 & 4.3 & 14.5 \\
None to accompany or care in hospital & 6.5 & 8.6 & 5.8 \\
Patient refused to be in hospital & 6.5 & 4.3 & 5.8 \\
Not sick or got cure & 0.0 & 8.7 & 2.9 \\
Total & 100.0 & 100.0 & 100.0 \\
\hline
\end{tabular}

$\mathrm{n}$ - number of deaths in each category; $\mathrm{X}^{2}($ with $\mathrm{df}=6)=14.8, P=0.02$

Table 6. Relationships (\%) of deceased who died in hospitals or in transit with decision makers of hospitalization, accompanying persons, and caregivers in hospitals

\begin{tabular}{lccc}
\hline $\begin{array}{l}\text { Relationships with the } \\
\text { deceased }\end{array}$ & $\begin{array}{c}\text { Decision maker of } \\
\text { hospitalization }(\mathbf{n}=\mathbf{8 5})\end{array}$ & $\begin{array}{c}\text { Hospital accompanying } \\
\text { person }(\mathbf{n}=\mathbf{8 5})\end{array}$ & $\begin{array}{c}\text { Caregivers in hospitals* } \\
(\mathbf{n}=\mathbf{7 4})\end{array}$ \\
\hline Son or daughter & 58.8 & 57.6 & 56.8 \\
Spouse & 17.6 & 16.5 & 24.3 \\
Parents & 8.2 & 9.4 & 8.1 \\
Patient self & 8.2 & 0.0 & 0.0 \\
Brother or sister & 0.0 & 8.2 & 5.4 \\
Health provider/worker & 4.7 & 0.0 & 0.0 \\
Grandparent/uncle & 0.0 & 8.2 & 1.4 \\
Other (relative/friend) & 2.3 & 0.0 & 4.0 \\
\hline
\end{tabular}

$\mathrm{n}$ - number of deaths in each category

*11 died on the way to hospitals, as such care in hospital was not applicable to them.

\section{PATIENTS DIED IN HOSPITALS OR ON THE WAY TO HOSPITAL: MAKING DECISIONS FOR HOSPITALIZATION}

Though making decisions is the domain of the healthcare providers, and the providers themselves participated in discussion about hospitalization, they were not commonly the final decision-makers (5\%) (Table 6). The most common final decision-makers for taking the patients to a hospital were sons and daughters (59\%), followed by spouse (18\%), patients themselves (8\%) and parents (8\% for young adult patients). The patients were accompanied to the hospital and cared for most often by sons and daughters (58\%), followed by spouses (17\%). Young adult patients were accompanied to hospitals by, and received care from, their parents. The average number of caregivers during a hospital stay was 1.9 persons. After recognizing an illness as severe and deciding to shift to a hospital, the patients were taken to hospitals primarily for cure of illness (93\%), followed by 'illness was too serious to provide care at home' (56\%), and patient's request (14\%) (Table 7). Patients were rarely hospitalized for palliative care (5\%).

\section{CHOICE OF A HEALTHCARE PROVIDER OR HOSPITAL AND DELAY IN HOSPITALIZATION}

Making decisions for hospitalization is followed by the choice of a healthcare provider or hospital for treatment. Factors considered in making choice of a hospital were: the quality of care (59\%), followed by short distance to the hospital (36\%), known doctors (35\%), referred by a doctor (29\%) or previous visit to the same hospital (15\%) (Table 7), Less frequently mentioned were costs of transportation (9\%) and hospital stay (7\%).

Upon inquiry about the time taken to shift the patients to a hospital after recognizing terminal illness or conditions, one in four patients was taken to a hospital after 24 hours of recognizing the illness. In the caregiver's view, there was a delay in taking the patients to a hospital in $42 \%$ cases and the percent of delay did not vary between age and sex groups (Table 8). The reasons stated for delay were misjudgment of severity of illness (31\%), lack of money and transport to shift (33\% each), or no male person present at home to accompany the patient (31\%). More than one source was used for payment of hospital bills - sources included family income and savings (87\% cases), followed by interest free loan from relatives or friends (46\%). In a few cases, payments were made from money borrowed from moneylen- 
Table 7. Distribution of factors that triggered hospitalization and items considered for choosing hospitals for deceased who died in hospitals or in transit

\begin{tabular}{lclc}
\hline $\begin{array}{l}\text { Triggering factors } \\
\text { for hospitalization }\end{array}$ & $\begin{array}{c}\text { \% } \\
(\mathbf{n = 8 5})\end{array}$ & $\begin{array}{l}\text { Items* considered for } \\
\text { choosing hospitals }\end{array}$ & $\begin{array}{c}\% \\
(\mathbf{n = 8 0 ) \dagger}\end{array}$ \\
\hline Hope for cure of illness & 92.9 & Quality of care & 58.7 \\
Too serious illness to care at home & 56.5 & Hospital is nearby & 36.2 \\
Patient's request & 14.1 & Doctor is known & 35.0 \\
Palliative care & 4.7 & Doctor's referral & 28.7 \\
& & Previous visit & 15.0 \\
& & Transport cost & 8.7 \\
\hline
\end{tabular}

$\mathrm{N}$ - number of deaths in each category

*Multiple response allowed.

$\nmid$ Not stated in 3 cases of ischemic heart disease and in 2 cases of stroke.

Table 8. Reasons for delay in hospitalization after making decisions, and payment of hospital costs for deceased who died in hospitals or in transit

\begin{tabular}{lrlr}
\hline Delay in shifting to a hospital (42\% of $\mathbf{8 5})$ & Payment* of hospital costs & \% ( $\mathbf{n}=\mathbf{8 0})+$ \\
Reasons* for delay & 30.6 & Family income and savings & 87.5 \\
\hline Misjudged severity of illness & 33.3 & Borrow from relatives or friends & 46.2 \\
Economic constraint & 33.3 & & \multirow{2}{*}{6.2} \\
No transport to shift & 19.4 & Borrow from moneylender or microcredit & 3.7 \\
Hospital at too far & 30.6 & Assets mortgage or sale & 1.2 \\
Male person not available & 8.3 & Health insurance & 1.2 \\
Others (doctor absent, rain, strike) & 5.6 & Other & \\
Patient afraid of surgical operation & & &
\end{tabular}

$\mathrm{n}$ - number of deaths in each category

*Multiple response allowed.

$\dagger$ Not stated in 3 cases of ischemic heart disease and in 2 cases of stroke.

ders on interest (6\%) or mortgage of assets (4\%). As expected, none except one was covered by health insurance.

\section{DISCUSSION}

The study revealed how healthcare decisions were made and implemented for terminally ill adult and elderly patients, and how treatment-related costs were managed at a household level in the study population. A great majority (85\%) of the patients died as a direct or indirect consequence of NCDs with a percentage being higher (93\%) in elderly age group. Some NCDs such as diabetes, hypertension, asthma, etc. are incurable, but are controllable to an extent through appropriate medication and behaviour change. The elderly patients are at increased risk for treatment failure in management of illness. ${ }^{20,21}$ Very old patients experiencing nonresponse to treatment sometimes refuse to be in hospital. People in rural areas particularly deem that illness in old age is not completely curable and sometimes even incurable when their illnesses do not respond to the treatments. ${ }^{17}$

These conceptions paired with economic hardship explain why $85 \%$ of the adult and elderly deaths registered in HDSS area in 2013 occurred at home. These terminally ill patients were treated at home for three major reasons: no chance of cure of illness, financial constraints, or misjudgment of illness severity; or were discharged if admitted from hospitals prior to recovery or death for two major reasons: no chance of cure or financial constraints. They were visited by both qualified and unqualified providers of modern medicine, as well as providers of traditional medicines in the course of illness for cure. These providers, during visits to the patients, briefed the caregivers on the health condition of the patients as well as the next course of treatment, chances of cure and treatment-related costs. The caregivers, noting no visible recovery of illness and treatment provider's opinion of no or little chance of cure, made the decision of not taking the patients to a hospital or returning from the hospital (if already admitted).

Financing healthcare from one's own pocket is one of the crucial deciding factors of whether or not to seek treatment, and what type of treatment to seek. In our study, financial constraints were the second major reason the caregivers stated for not seeking treatment, seeking treatment from untrained providers, delaying treatment, not taking the patient to a hospital, or taking back the patients from hospitals prior to death even after recognizing the illness as severe. This was evident in the payment of hospital bills by taking loans from relatives (46\%), moneylenders (6\%) or asset mortgages (4\%). This finding echoes the delay in 
seeking treatment from well-trained providers for high cost of services in Bangladesh and Uganda. ${ }^{22,23}$ The high cost of formally trained allopathic physicians was generally the reason they were avoided, unless high severity demanded them. ${ }^{17,24}$ Due to financial constraints, some of the terminally ill patients who had a reasonable chance of cure were not taken to a well-trained provider or a hospital for treatment. If the patients had received timely treatment, it is likely that they would have survived.

Early detection and recognition of illness severity is an important step towards seeking care. An ethnographic study in Ghana found that mothers were not able to recognize serious illness in their children. ${ }^{25}$ Poor knowledge of danger signs can result in misjudgment of the severity of illness and can delay care-seeking. In our study, the third major reason for not shifting the patients who later died at home to a hospital, or for delaying in shifting the patients to a hospital after making decisions, was the misjudgment of the illness severity. This has also been reported for female patients of reproductive age in Bangladesh, and for severe maternal morbidity in Uganda. ${ }^{22,23}$

Health-seeking depends on the perception of health and ill-health, and therefore the judgment of illness severity. This is especially apparent in the elderly, as they suffer from chronic illnesses that occasionally deteriorate to acute illnesses urgently requiring medical attention. The elderly might have had similar acute conditions and manifestations of chronic diseases before, and caregivers might have overseen the condition and management of the illnesses. If this were the case, caregivers might have adopted a regimen, and perceived the severity of illness as low. If an illness is regarded as having low severity, treatment from trained providers is denied or delayed. Therefore, both the patients and the caregivers need to know when to adopt home care to assuage suffering and when to forgo home care for immediate professional care. Imparting knowledge of danger signs and limitations of home care may change the situation.

Participants in discussions for managing the illnesses of patients included family members, patients, relatives, friends and health providers. However, the final decision to take the patients to a hospital and choice of the hospital were made by family members, even though choice of medical care and where to take the patient should be within the domain of a physician in order to yield the best possible results and meet patient needs. Choice by a physician facilitates the optimal utilization of healthcare resources, often under challenging and imperfect circumstances. A household's ability to pay out-of-pocket medical costs may limit the role of the physician in the choice of medical care, so it is ultimately the family member who makes the final decision. In Kenya, fathers are the main income earners and thus make the final decision concerning where the child is taken for treatment. ${ }^{16}$

Usually, good quality of care and reasonable cost persuade the family member to choose hospitals for treatment. While distance to hospital is commonly suggested as a factor in health facility use, women in India have been found to travel further to attend private, more expensive services perceived to be of 'good quality'. ${ }^{26}$ In Sri Lanka and Burkina Faso, caregivers often bypass the nearest health facility when seeking medical care, and take the sick person to facilities with better reputations for quality of care relative to cost. ${ }^{27,28}$ Similar to the other studies, our study revealed quality of care as a major factor in choosing a hospital. Other factors such as distance, familiarity with doctors and doctor's referral also influenced the choice of hospital. Cost of services and the waiting time were important factors in choosing a health facility in south western Nigeria. ${ }^{29}$

The study findings may suffer from a number of limitations. Selection of the sample for studying decision-making of the patients who died at home and who died in hospitals was not an age-sex matched sample, and the illness history and care-seeking information were based on interviews by non-medical personnel. Interviews involving a long recall period may suffer lower reliability and validity of the findings. Since the study was done in one specific area, the findings might not be generalizable for the entirety of rural Bangladesh. However, much of rural Bangladesh is homogeneous with respect to economic activity, language, culture, social norms and public health systems, and the findings thus give a fair idea about the rest of the country. Decision-making for health-seeking can be different for acute and chronic conditions, and for males and females, depending on the role of the husband, social networks and cultural customs, but these were not separately estimated because of the small sample size. Moreover, the study sample included few sudden deaths, namely persons dying without prior disease signs and symptoms, requiring healthcareseeking. This factor may have resulted in an underestimation of the levels of care-seeking. Despite these limitations, this study used social autopsy - instead of the use of health services - to gauge how rural people respond to illness. The study identified the major reasons for not shifting patients who died at home to a hospital, or reasons for hospital discharge if the patients were shifted during terminal illness prior to recovery or death, as well as reasons for delay in hospitalization.

In the study area, $15 \%$ of the adult and elderly deaths in 2013 occurred in hospitals or in-transit. This low rate of hospital deaths could be the outcome of psychosocial perceptions related to the patients' chance of recovery, inability to judge severity of illness, and economic constraints in accessing health services. Local-level and national-level advocacy can change the psychosocial perceptions related to the social notion that 'old-age is ill-health' and judgment of illness severity. The resource-poor rural areas need practical healthcare financing to lower out-of-pocket expenditures for professional care of illness. Improving family members' ability to recognize severity of illness, as well as reducing financial constraints, may increase affected persons' chances of receiving reasonable care in a hospital and overall chances of survival.

\section{ACKNOWLEDGEMENTS}

This research study was funded by National Health and Medical Research Council, Australia through University of Queensland School of Public Health and icddr,b. Current donors, which provide unrestricted support to icddr,b for its 
operation and research include: the Government of the People's Republic of Bangladesh, Global Affairs Canada (GAC), Swedish International Development Cooperation Agency (SIDA) and the Department for International Development (UK Aid). These donors are gratefully acknowledged for their support and commitment to icddr,b's research efforts.

\section{FUNDING}

This study has been funded by a project grant (grant \# GR-00885) of National Health and Medical Research Council, Australia; however, it had no role in study design, data collection and analysis, decision to publish or preparation of the manuscript.

\section{COMPETING INTERESTS}

The authors completed the Unified Competing Interest form at http://www.icmje.org/coi_disclosure.pdf (available upon request from the corresponding author), and declare no conflicts of interest.

\section{CORRESPONDENCE TO:}

Nurul Alam

Health Systems and Population Studies Division

International Centre for Diarrhoeal Disease Research, Bangladesh

Mohakhali

Dhaka 1212

Bangladesh

nalam@icddr,b.org 


\section{REFERENCES}

1. Källander K, Kadobera D, Williams TN, et al. Social autopsy: INDEPTH Network experiences of utility, process, practices, and challenges in investigating causes and contributors to mortality. Popul Health Metrics. 2011;9(1):44. doi:10.1186/1478-7954-9-44

2. Kalter HD, Salgado R, Babille M, Koffi AK, Black RE. Social autopsy for maternal and child deaths: a comprehensive literature review to examine the concept and the development of the method. Popul Health Metrics. 2011;9(1):45. doi:10.1186/1478-795 4-9-45

3. Chapman GB, Sonnenberg FA. Decision-Making in Health Carehealthcare: Theory, Psychology, and Applications. Cambridge University Press; 2003.

4. van Doorslaer E, O’Donnell O, Rannan-Eliya RP, et al. Catastrophic payments for health care in Asia. Health Econ. 2007;16(11):1159-1184. doi:10.1002/he c. 1209

5. Ministry of Health and Family Welfare. Bangladesh National Health Accounts 1997 - 2012. Health Economics Unit, Ministry of Health and Family Welfare, Government of the People's Republic of Bangladesh; 2015.

6. Tahsina T, Ali NB, Hoque DME, et al. Out-of-pocket expenditure for seeking health care for sick children younger than 5 years of age in Bangladesh: findings from cross-sectional surveys, 2009 and 2012. J Health Popul Nutr. 2017;36(1). doi:10.1186/s41043-017-011 $\underline{0-4}$

7. Alam N, Chowdhury HR, Bhuiyan MA, Streatfield PK. Causes of death of adults and elderly and healthcare-seeking before death in rural Bangladesh. J Health Popul Nutr. 2010;28(5):520-528. doi:10.3329/j hpn.v28i5.6161

8. Alam N, Chowdhury HR, Das SC, Ashraf A, Streatfield PK. Causes of death in two rural demographic surveillance sites in Bangladesh, 2004-2010: automated coding of verbal autopsies using InterVA-4. Glob Health Action. 2014;7(1):25511. doi:10.3402/gha.v7.25511

9. El-Saharty S, Ahsan KZ, Koehlmoos TLP, Engelgau MM. Tackling Noncommunicable Diseases in Bangladesh: Now Is the Time. The World Bank; 2013. $\underline{\mathrm{d}}$ oi:10.1596/978-0-8213-9920-0
10. Leslie HH, Spiegelman D, Zhou X, Kruk ME. Service readiness of health facilities in Bangladesh, Haiti, Kenya, Malawi, Namibia, Nepal, Rwanda, Senegal, Uganda and the United Republic of Tanzania. Bull World Health Organ. 2017;95(11):738-748. doi:10.2471/blt.17.191916

11. Moucheraud C. Service Readiness For Noncommunicable Diseases Was Low In Five Countries in 2013-15. Health Aff. 2018;37(8):1321-1330. doi:10.1377/hlthaff.2018.0151

12. National Institute of Population Research and Training, Associates for Community and Population Research, ICF International. Bangladesh Health Facility Survey 2014. NIPORT, ACPR, and ICF International; 2016.

13. Subramanian S, Gakunga R, Kibachio J, et al. Cost and affordability of non-communicable disease screening, diagnosis and treatment in Kenya: Patient payments in the private and public sectors. PLoS ONE. 2018;13(1):e0190113. doi:10.1371/journal.pon e. 0190113

14. Pryer J. When breadwinners fall ill: Preliminary findings from a case study in Bangladesh. IDS Bull. 1989;20(2):49-57. doi:10.1111/j.1759-5436.1989.mp2 0002007.x

15. Alvesson HM, Lindelow M, Khanthaphat B, Laflamme L. Shaping healthcare-seeking processes during fatal illness in resource-poor settings. A study in Lao PDR. BMC Health Serv Res. 2012;12(1):477. do i:10.1186/1472-6963-12-477

16. Abubakar A, Van Baar A, Fischer R, Bomu G, Gona JK, Newton CR. Socio-cultural determinants of health-seeking behaviour on the Kenyan coast: a qualitative study. PLoS ONE. 2013;8(11):e71998. doi:1 0.1371/journal.pone.0071998

17. Biswas P, Kabir ZN, Nilsson J, Zaman S. Dynamics of health care seeking behaviour of elderly people in rural Bangladesh. Int J Ageing Later Life. 2006;1(1):69-89. doi:10.3384/ijal.1652-8670.061169

18. icddr b. Health and Demographic Surveillance System - Matlab: Registration of Health and Demographic Events 2013. Scientific Report No.126; 2015.

19. icddr b. Institutional Review Board: List of completed protocols 31 July 2018. Accessed November 15, 2017. http://shetu.icddrb.org/index.php?option $=$ c om_content \&view=article \&id=272 \&Itemid=684 
20. Haran JP, Wilsterman E, Zeoli T, Beaudoin FL, Tjia J, Hibberd PL. Elderly patients are at increased risk for treatment failure in outpatient management of purulent skin infections. Am J Emerg Med.

2017;35(2):249-254. doi:10.1016/j.ajem.2016.10.060

21. London S. Risk for Asthma Treatment Failure Rises With Age. Published 2015. Accessed August 14, 2018. https://www.medscape.com/viewarticle/846468

22. Sikder SS, Labrique AB, Ullah B, et al. Careseeking patterns for fatal non-communicable diseases among women of reproductive age in rural northwest Bangladesh. BMC Womens Health. 2012;12(1):23. do i:10.1186/1472-6874-12-23

23. Okong P, Byamugisha J, Mirembe F, Byaruhanga $\mathrm{R}$, Bergstrom S. Audit of severe maternal morbidity in Uganda - implications for quality of obstetric care. Acta Obstet Gynecol Scand. 2006;85(7):797-804. doi:1 $\underline{0.1080 / 00016340600593331}$

24. Ahmed SM, Tomson G, Petzold M, Kabir ZN. Socioeconomic status overrides age and gender in determining health-seeking behaviour in rural Bangladesh. Bull World Health Organ. 2005;83:109-117.
25. Bazzano AN, Kirkwood BR, Tawiah-Agyemang C, Owusu-Agyei S, Adongo PB. Beyond symptom recognition: care-seeking for ill newborns in rural Ghana. Trop Med Int Health. 2008;13(1):123-128. do i:10.1111/j.1365-3156.2007.01981.x

26. Bhatia JC, Cleland J. Health-care seeking and expenditure by young Indian mothers in the public and private sectors. Health Policy Plan.

2001;16(1):55-61. doi:10.1093/heapol/16.1.55

27. Akin JS, Hutchinson P. Health-care facility choice and the phenomenon of bypassing. Health Policy Plan. 1999;14(2):135-151. doi:10.1093/heapol/14.2.135

28. Sauerborn R, Nougtara A, Diesfeld HJ. Low utilization of community health workers: results from a household interview survey in Burkina Faso. Soc Sci Med. 1989;29(10):1163-1174. doi:10.1016/0277-953 6(89)90359-6

29. Uchendu OC, Ilesanmi O, Olumide A. Factors influencing the choice of health care providing facility among workers in a local government secretariat in south western Nigeria. Ann Ib Postgrad Med. 2013;11:87-95. 